\title{
Water Extract of Deer Bones Activates Macrophages and Alleviates Neutropenia
}

\author{
Han-Seok Choi, Soon Re Kim, Se Hyang Hong, Jin Mo Ku, Min Kyoung Kim, \\ Hye Sook Seo, Sung-Gook Cho, Sangtae Shin, Yong Cheol Shin, and Seong-Gyu Ko \\ Department of Preventive Medicine, College of Korean Medicine, Kyung Hee University, Seoul 130-701, Republic of Korea \\ Correspondence should be addressed to Seong-Gyu Ko; epiko@khu.ac.kr
}

Received 4 April 2013; Accepted 11 May 2013

Academic Editor: Bo-Hyoung Jang

Copyright (C) 2013 Han-Seok Choi et al. This is an open access article distributed under the Creative Commons Attribution License, which permits unrestricted use, distribution, and reproduction in any medium, provided the original work is properly cited.

\begin{abstract}
Extracts from deer bones, called nok-gol in Korean, have long been used to invigorate Qi. While neutropenia is not well detected in normal physiological condition, it could be a cause of severe problems to develop diseases such as infectious and cancerous diseases. Thus, a prevention of neutropenia in normal physiology and pathophysiological states is important for maintaining Qi and preventing disease progress. In cell biological aspects, activated macrophages are known to prevent neutropenia. In this study, we demonstrate that water extract of deer bone (herein, NG) prevents neutropenia by activating macrophages. In mouse neutropenia model system in vivo where ICR mice were treated with cyclophosphamide to immunosuppress, an oral administration of NG altered the number of blood cells including lymphocytes, neutrophils, basophils, and eosinophils. This in vivo effect of NG was relevant to that of granulocyte colony stimulating factor (G-CSF) that was known to improve neutropenia. Our in vitro studies further showed that NG treatment increased intracellular reactive oxygen species (ROS) and promoted macrophagic differentiation of mouse monocytic Raw264.7 cells in a dose-dependent manner. In addition, NG enhanced nitric oxide (NO) synthesis and secretions of cytokines including IL- 6 and TNF- $\alpha$. Consistently, NG treatment induced phosphorylation of ERK, JNK, IKK, I $\kappa \mathrm{B} \alpha$, and NF- $\kappa$ B in Raw264.7 cells. Thus, our data suggest that NG is helpful for alleviating neutropenia.
\end{abstract}

\section{Introduction}

Neutropenia is characterized by a low neutrophil number, which could be a risk of disease development including infectious and cancerous diseases [1-6]. Thus, therapeutic approaches such as administration with G-CSF or GMCSF have been applied to prevent neutropenic phenomenon, while no trials are completely worked yet [7-10]. Macrophages, one of the players in the innate immune system, modulate immune response through inflammatory cytokines and reactive oxygen species (ROS) [11-13]. In neutropenic situation, macrophages could not be involved in inflammation because neutrophil depletion results in no reaction of monocytes and macrophages [14]. As macrophages produce CSF, an activation of macrophages is crucial for preventing neutropenia and secondary diseases [15-17].

Extracts from deer bones, called nok-gol in Korean, have been traditionally used as a drug invigorating Qi. Recent researches further reported various therapeutic effects of this traditional medicine on inflammatory disease $[18,19]$, bone resorption [20], and aging [21]. Furthermore, extracts of deer bones affected macrophagic activation in S. aureus-infected mice [22]. In this study, we demonstrate that water extract of deer bones (hereafter, NG) alleviates neutropenia through activating macrophages.

\section{Materials and Methods}

2.1. Cell Culture and Deer Bone Extract Preparation. Raw264.7 mouse monocytic/macrophagic cells were grown in Dulbecco's Modified Eagle's Medium (DMEM) supplemented with $10 \%$ heat-inactivated fetal bovine serum (FBS) and $1 \%$ antibiotics at $37^{\circ} \mathrm{C}$ in a $5 \% \mathrm{CO}_{2}$ humidified incubator. Water extract of deer bones (NG) was provided by Nongshim Corporation (Republic of Korea). More information on NG production processes could be requested from the company. 
TABLE 1: The sequence of PCR primers.

\begin{tabular}{|c|c|}
\hline Primer name & Sequences \\
\hline \multicolumn{2}{|l|}{ IL-6 } \\
\hline Forward & $5^{\prime}$-CAA GAG ACT TCC ATC CAG TTG C-3' \\
\hline Reverse & $5^{\prime}$-TTG CCG AGT TCT CAA AGT GAC-3' \\
\hline \multicolumn{2}{|l|}{ TNF- $\alpha$} \\
\hline Forward & $5^{\prime}$-ATG AGC ACA GAA AGC ATG ATC-3' \\
\hline Reverse & $5^{\prime}$-TAC AGG CTT GTC ACT GGA ATT-3' \\
\hline \multicolumn{2}{|l|}{ COX-2 } \\
\hline Forward & $5^{\prime}$-AAG ACT TGC CAG GCT GAA CT- $3^{\prime}$ \\
\hline Reverse & $5^{\prime}$-CTT CTG CAG TCC CAG GTT CAA- $3^{\prime}$ \\
\hline \multicolumn{2}{|l|}{ iNOS } \\
\hline Forward & $5^{\prime}$-AAT GGC AAC ATC AGG TCG GCC ATC ACT-3' \\
\hline Reverse & $5^{\prime}$-GCT GTG TGT CAC AGA AGT CTC GAA CTC- ${ }^{\prime}$ \\
\hline \multicolumn{2}{|l|}{ GAPDH } \\
\hline Forward & $5^{\prime}$-GAG GGG CCA TCC ACA GTC TTC-3' \\
\hline Reverse & $5^{\prime}$-CAT CAC CAT CTT CCA GGA GCG-3' \\
\hline
\end{tabular}

TABLE 2: The number of WBC, lymphocytes, neutrophils, monocytes, and eosinophils in mice blood. Each value presents the mean \pm SD $\left({ }^{*} P<0.05,{ }^{* *} P<0.01\right.$, and $\left.{ }^{* * *} P<0.001\right)$.

(a)

\begin{tabular}{lcccc}
\hline & Normal & Negative Con & Positive Con & NG \\
\hline WBC (K/uL) & $10.89 \pm 1.57$ & $2.49 \pm 1.48^{* * *}$ & $4.93 \pm 0.71^{* * *}$ & $5.10 \pm 1.48^{* *}$ \\
Lymphocytes (K/uL) & $6.21 \pm 1.27$ & $0.76 \pm 0.27^{* * *}$ & $1.77 \pm 0.39^{* *}$ & $2.00 \pm 0.56^{* *}$ \\
Neutrophils (K/uL) & $2.81 \pm 0.77$ & $1.07 \pm 0.72^{*}$ & $2.75 \pm 0.67$ & $2.91 \pm 0.79$ \\
Monocytes (K/uL) & $0.59 \pm 0.16$ & $0.18 \pm 0.07^{* *}$ & $0.15 \pm 0.07^{* * *}$ & $0.12 \pm 0.04^{* *}$ \\
Eosinophils (K/uL) & $0.22 \pm 0.07$ & $0.37 \pm 0.06^{*}$ & $0.38 \pm 0.06^{*}$ & $0.30 \pm 0.13$ \\
Basophils (K/uL) & $0.05 \pm 0.02$ & $0.04 \pm 0.02$ & $0.05 \pm 0.02$ & $0.07 \pm 0.04$ \\
\hline
\end{tabular}

(b)

\begin{tabular}{|c|c|c|c|c|}
\hline & Normal & Negative Con & Positive Con & NG \\
\hline Lymphocytes (\%) & $60.01 \pm 6.85$ & $41.93 \pm 9.20^{*}$ & $41.64 \pm 9.18^{*}$ & $31.05 \pm 7.25^{* * *}$ \\
\hline Neutrophils (\%) & $29.87 \pm 8.26$ & $35.15 \pm 19.12$ & $55.95 \pm 11.12^{* *}$ & $60.31 \pm 7.88^{* * *}$ \\
\hline Monocytes (\%) & $5.59 \pm 0.64$ & $5.21 \pm 1.97$ & $6.77 \pm 1.62$ & $4.06 \pm 2.73$ \\
\hline Eosinophils (\%) & $2.79 \pm 1.33$ & $8.99 \pm 2.58^{* *}$ & $7.12 \pm 2.04^{* *}$ & $4.68 \pm 0.73^{*}$ \\
\hline Basophils (\%) & $0.50 \pm 0.10$ & $1.78 \pm 0.89^{*}$ & $1.54 \pm 0.23^{* * *}$ & $1.07 \pm 0.62$ \\
\hline
\end{tabular}

2.2. In Vivo Studies. Six-week-old male ICR mice were purchased from Orient (Sungnam, Republic of Korea). All procedures were performed on the approval of the animal care center of Kyung Hee University (Approval no. KHUASP (SE)12-042). To induce neutropenia, mice were intraperitoneally injected with $150 \mathrm{mg} / \mathrm{kg}$ of cyclophosphamide twice in one week and then treated with cyclophosphamide $(100 \mathrm{mg} / \mathrm{kg})$ two times in another week. Mice were rested for one week with no treatment when treatments were switched. In addition, mice were randomized into 4 groups (normal, control, G-CSF, and NG; five mice per group) during resting time points. G-CSF $(1 \mu \mathrm{g} / \mathrm{kg})$ or NG $(200 \mathrm{mg} / \mathrm{kg})$ was orally administered every day for the last procedure. At the end of the experiment, mice were sacrificed by $\mathrm{CO}_{2}$ inhalation, and cardiac blood was collected.
2.3. Cell Surface Observation. Cells seeded into $60 \mathrm{~mm}$ culture dish at a density of $3 \times 10^{5}$ cells/dish were treated with NG $(25,250,500 \mu \mathrm{g} / \mathrm{mL})$ for $24 \mathrm{~h}$. Cell surfaces were observed by taking images using a camera (Olympus, Japan) connected to a light microscope.

2.4. Cell Viability Assay. Cell viability was determined using WST assay (Dogen, Republic of Korea). RAW264.7 cells $(1 \times$ $10^{4}$ cells/well) were seeded into 96-well plates and incubated overnight. Cells were then treated with different concentrations of NG and incubated for another 24 hours. $10 \mu \mathrm{L}$ of WST solution was added to $100 \mu \mathrm{L}$ cell culture medium, and plates were incubated for 2 hours. Optical density (OD) was determined at $450 \mathrm{~nm}$ using a microplate reader (Versa Max, Molecular Devices, CA, USA). 


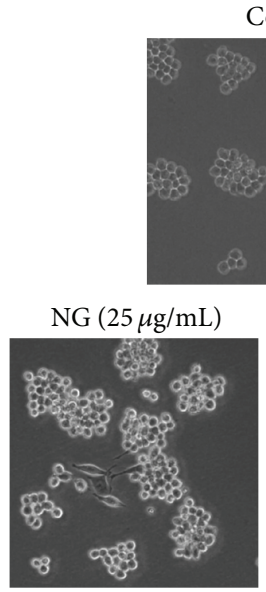

Con
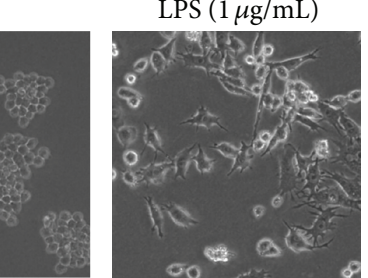

NG $(250 \mu \mathrm{g} / \mathrm{mL})$

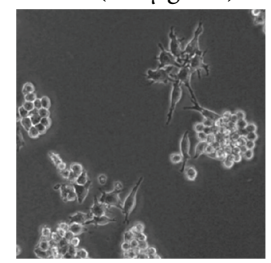

(a)

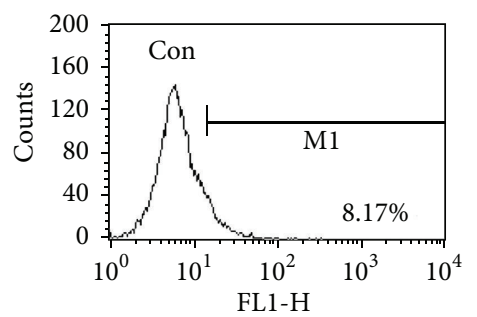

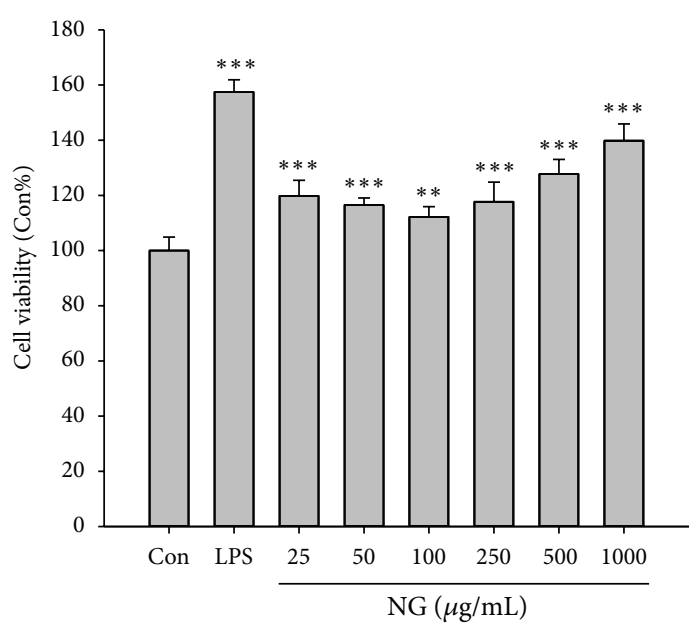

(b)
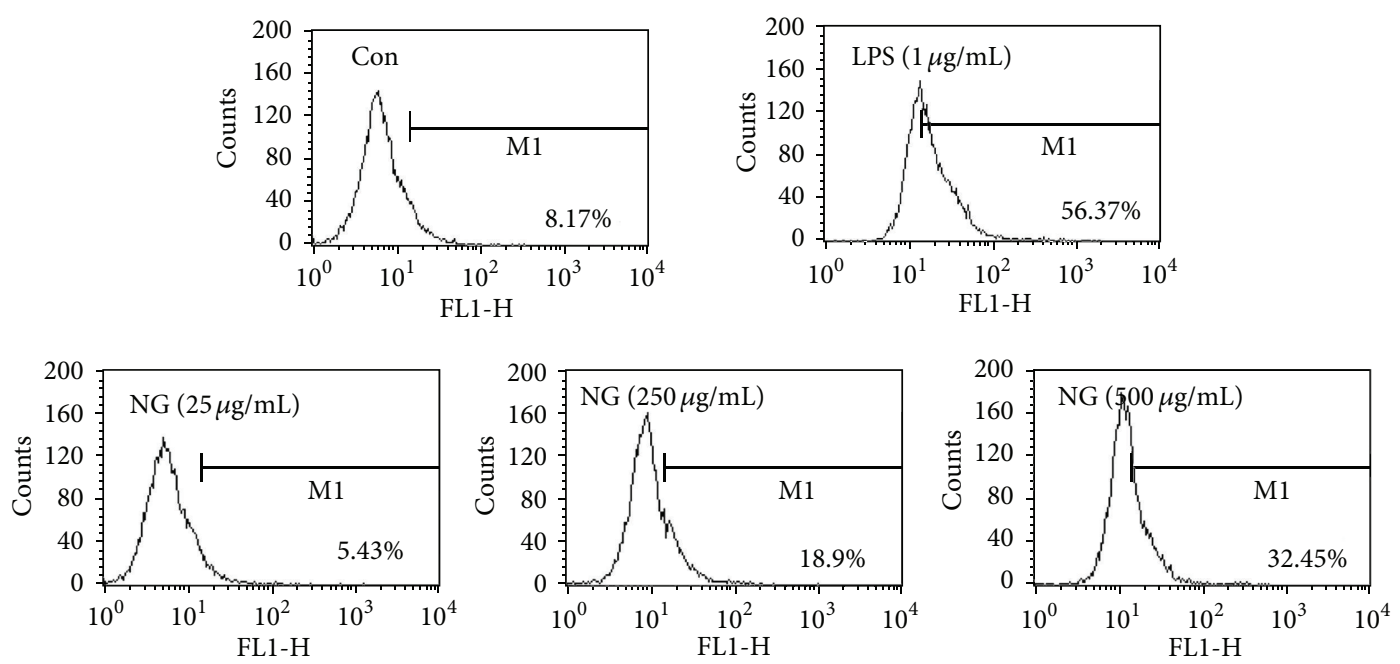

(c)

Figure 1: NG activates RAW264.7 cells. (a) Cells were seeded into $60 \mathrm{~mm}$ culture dish at a density of $3 \times 10^{5}$ cells/dish. The next day, cells were treated with NG for $24 \mathrm{~h}$. Cell surface was observed by taking a photograph using a camera attached to a microscope. (b) Cell viability was determined using WST assay after NG treatment. (c) ROS level was measured using DCFH-DA. Data are shown as the mean of three independent experiments (error bars are mean \pm standard deviation $(\mathrm{SD}))\left({ }^{*} P<0.05,{ }^{* *} P<0.01\right.$, and ${ }^{* * *} P<0.001$ ).

2.5. Intracellular ROS Level Measurement. Raw264.7 cell pellets were washed with PBS and incubated with $20 \mu \mathrm{M} \mathrm{DCFH-}$ $\mathrm{DA}$ for 1 hour at $37^{\circ} \mathrm{C}$ in the dark. After washing with PBS, green fluorescence ( $480 \mathrm{~nm}$ excitation $/ 530 \mathrm{~nm}$ emission) was measured by flow cytometry (BD FACSCalibur).

2.6. Detection of Nitric Oxide (NO). NO production was measured by adding Griess reagent in culture medium (Welgene, Republic of Korea). In detail, $150 \mu \mathrm{L}$ of supernatant from each well was transferred to 96-well plate and then mixed with $150 \mu \mathrm{L}$ of Griess reagent solution. Mixtures were then incubated for $30 \mathrm{~min}$ at room temperature. OD was determined at $570 \mathrm{~nm}$ using a microplate reader.

2.7. RT-PCR. Raw 264.7 cell pellets were washed with ice-cold $\mathrm{PBS}$, and RNAs were extracted using the easy-blue RNA extraction kit (Intron Biotech, Korea) according to the manufacturer's instructions. Total RNAs were quantified using NanoDrop ND-1000 spectrophotometer (NanoDrop Technologies Inc.) and subjected to reverse transcriptions using cDNA synthesis kit (TaKaRa, Japan). Conventional PCRs were then performed using appropriate primers. GAPDH was used as an internal control. Primer information was described in Table 1.

2.8. IL-6 and TNF- $\alpha$ Measurement. IL- 6 and TNF- $\alpha$ levels from Raw264.7 were measured by sandwich ELISA using BD Pharmingen mouse ELISA set. Cells in 6-well plates were treated with different concentrations of NG and incubated for $24 \mathrm{~h}$. Cytokine levels were measured at $450 \mathrm{~nm}$ using a microplate reader. 


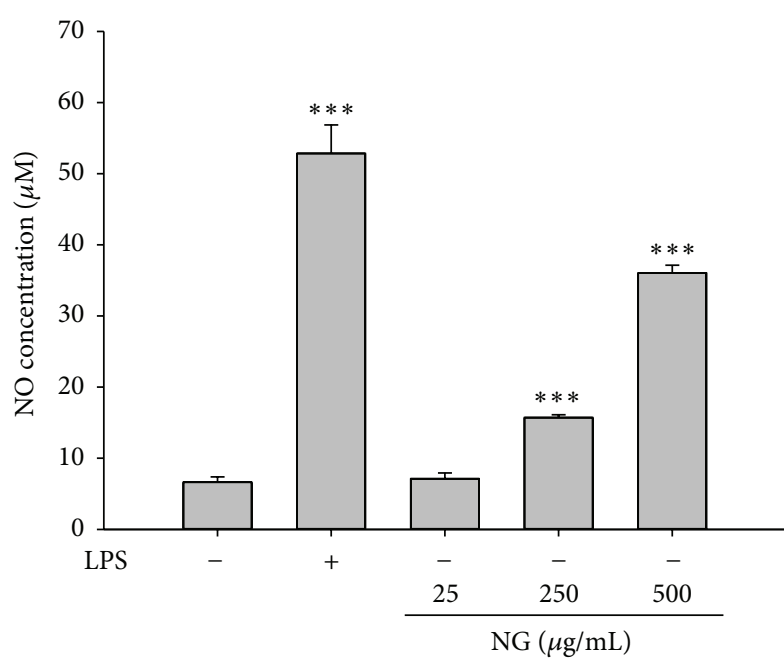

(a)

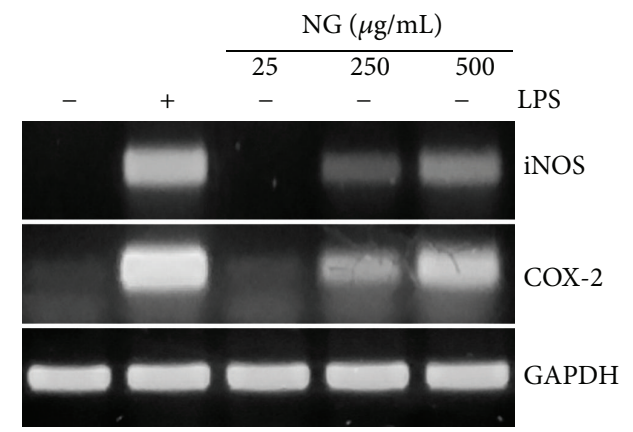

(b)

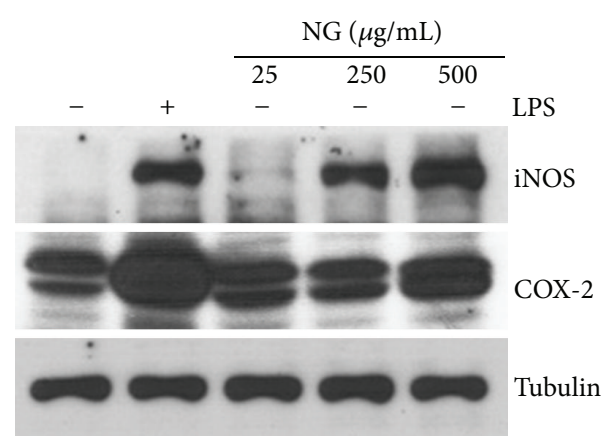

(c)

FIGURE 2: NG augmented NO concentration and iNOS and COX-2 expression. (a) NO production was measured using Griess reagent. (b) iNOS and COX-2 mRNA expression was measured by RT-PCR. (c) iNOS and COX-2 protein expression was measured by Western blot. Data are shown as the mean of three independent experiments (error bars are mean \pm standard deviation $(\mathrm{SD}))\left({ }^{*} P<0.05,{ }^{* *} P<0.01\right.$, and $\left.{ }^{* * *} P<0.001\right)$.

2.9. Western Blot Analysis. Whole cell lysates were washed with ice-cold PBS and lysed with RIPA buffer. Equal amount of protein $(30 \mu \mathrm{g})$ was run on $10 \%$ SDS-PAGE and transferred to nitrocellulose membranes. Blotted membranes were then incubated overnight at $4^{\circ} \mathrm{C}$ with appropriate primary antibodies. After washing in PBS-tween 20 for 1 hour, membranes were incubated with appropriate HRP-conjugated secondary antibodies and bands were visualized with the enhanced chemiluminescence detection system (AmershamPharmacia Biotech, Buckinghamshire, UK).

2.10. Analyses of Cardiac Blood. Whole blood samples from mice were collected by cardiac punctures. Blood was loaded in Vacutainer tubes containing EDTA (BD Biosciences, USA). Analyses of WBC, neutrophil, and monocyte in blood were done using blood analyzer (Hemavet 950, Drew Scientific, Germany).

2.11. Statistical Analysis. Data from experiments performed in triplicate presented mean \pm standard deviation. Statistical significance was obtained by Student's $t$-test, and a $P$ value less than 0.05 was considered statistically significant.

\section{Results and Discussion}

3.1. Water Extract of Deer Bone (NG) Induces RAW264.7 Cell Differentiation. LPS has been known to induce a differentiation of monocytic Raw264.7 cells to macrophages. Consistently, LPS $(1 \mu \mathrm{g} / \mathrm{mL})$ induced Raw264.7 differentiation in our experiment. Likewise, NG treatment also resulted in Raw264.7 cell differentiation in a dose-dependent manner (Figure 1(a)). In addition, both LPS and NG increased WST activities in RAW264.7 cells, indicating that NG induced activities of dehydrogenases (Figure 1(b)). Macrophages secrete ROS, when being activated [23]. So, we further measured ROS levels in Raw264.7 cells treated with different concentrations of NG or LPS. As seen in Figure 1(c), NG augmented ROS level in a dose-dependent manner. Thus, our data indicate that NG induces Raw264.7 cell differentiation to macrophages. 


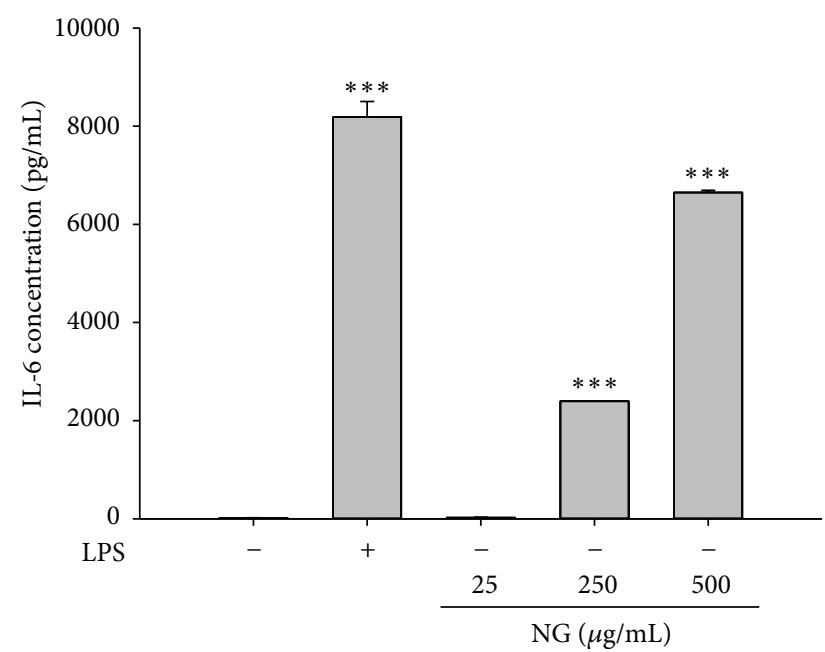

(a)

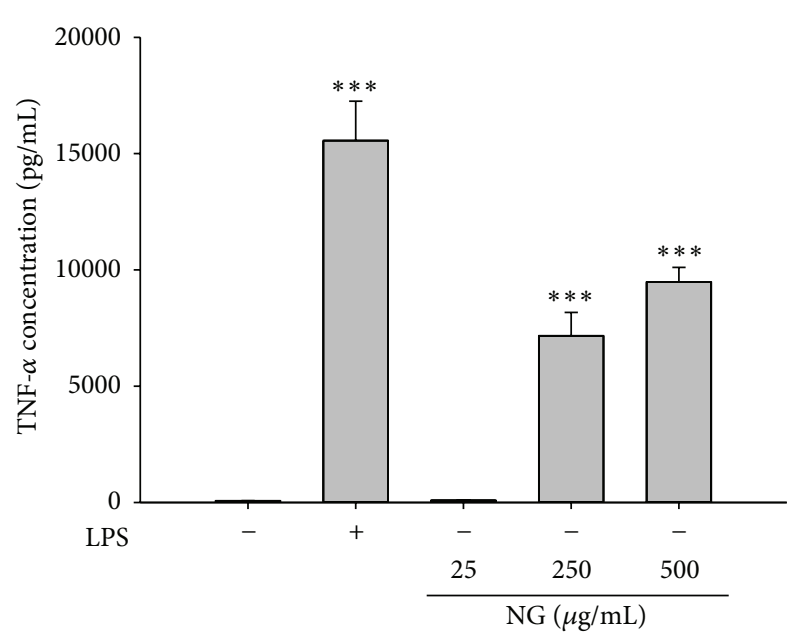

(b)

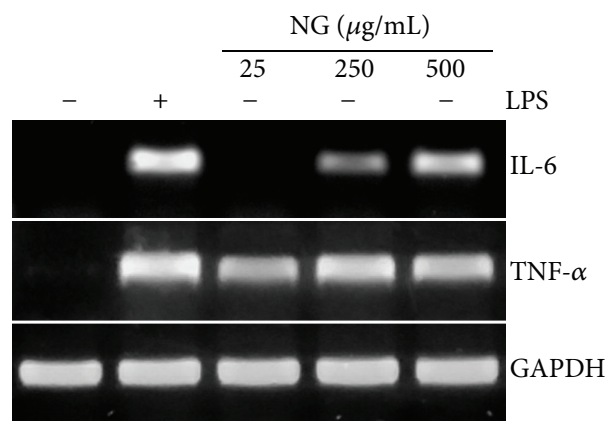

(c)

FIGURE 3: NG increased cytokines production. The release of IL- 6 and TNF- $\alpha$ was measured by sandwich ELISA assay. Data are shown as the mean of three independent experiments (error bars are mean \pm standard deviation (SD)) $\left({ }^{*} P<0.05,{ }^{* *} P<0.01\right.$, and $\left.{ }^{* * *} P<0.001\right)$.

3.2. NG Increases NO Concentration and Induces Expression of iNOS and COX-2. We next examined whether NG affects intracellular NO contents in Raw264.7 cells. When Raw264.7 cells were treated with different concentrations of NG or LPS as a positive control, both NG and LPS increased NO production (Figure 2(a)). As NO generation is associated with iNOS and COX-2, we further examined whether NG affects iNOS and COX-2. NG treatment increased expression of both iNOS and COX-2 (Figures 2(b) and 2(c)).

3.3. NG Produces $I L-6$ and TNF- $\alpha$. Macrophages react to microbial invasion through IL- 6 and TNF- $\alpha$ secretion $[17$, 24]. Therefore, we measured the levels of IL- 6 and TNF- $\alpha$ in Raw264.7 cells treated with different concentrations of NG for 24 hours. NG as well as LPS significantly increased levels of IL-6 and TNF- $\alpha$ (Figures 3(a) and 3(b)). This NG effect on IL- 6 and TNF- $\alpha$ resulted from transcriptional regulation of both cytokines because NG treatment induced mRNA expression of either IL-6 or TNF- $\alpha$ (Figure 3(c)).

3.4. NG Induces Activation of MAPKs and NF- $\kappa B$. It has been reported that LPS activates macrophages through activation of MAPKs and NF- $\kappa$ B [25]. So, we examined whether NG affects signaling pathways of MAPKs and NF- $\kappa$ B in Raw264.7 cells. When Raw264.7 cells were treated with $250 \mu \mathrm{g} / \mathrm{mL}$ of NG for 360 minutes, ERK phosphorylation was increased at 10 minutes after NG treatment and sustained until 30 minutes after NG treatment. JNK phosphorylation was increased at 10 minutes after NG treatment and peaked up at 30 minutes. However, NG appeared not to affect p38 phosphorylation (Figure $4(\mathrm{a})$ ). We also found that NG activates NF- $\kappa \mathrm{B}$ pathway. IKK phosphorylation was gradually increased from 10 minutes and peaked up at 30 minutes after NG treatment. Likewise, $\mathrm{I} \kappa \mathrm{B}$ phosphorylation was increased during that time points and total $\mathrm{I} \kappa \mathrm{B}$ level was consistently reduced (Figure 4(b)). Accordingly, NG resulted in nuclear accumulation of phosphorylated form of NF- $\kappa \mathrm{B}$, when phosphorylated form of NF- $\kappa \mathrm{B}$ in cytoplasmic and nuclear fractions were analyzed (Figure 4(c)). Thus, our data indicate that NG activates MAPK and NF-kB pathways as like LPS (Figure 5).

3.5. NG Alleviates in Mouse Neutropenia Model System. To verify NG effect in immune systems in vivo, we applied mouse neutropenia model system. In mice treated with NG, blood analyses presented a decrease of lymphocyte numbers and an increase of either neutrophils or eosinophils (Table 2). In NGtreated mice compared to normal mice, lymphocyte numbers 


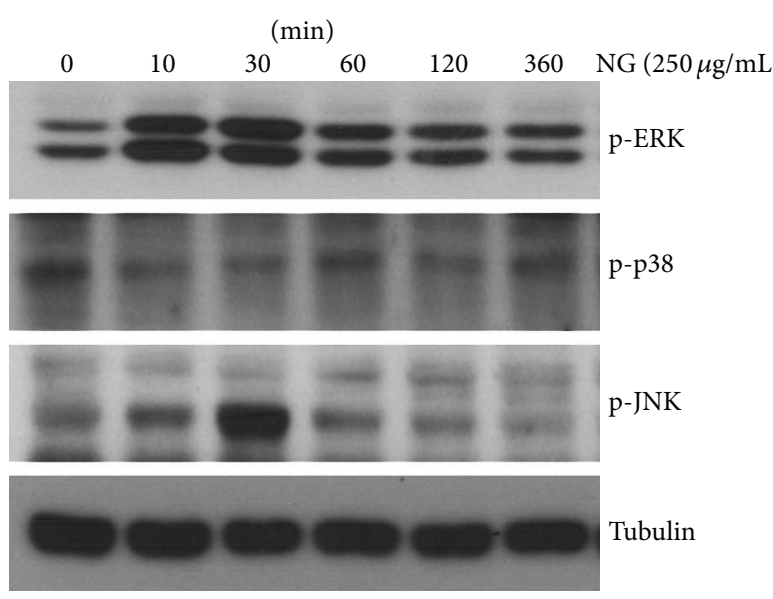

(a)
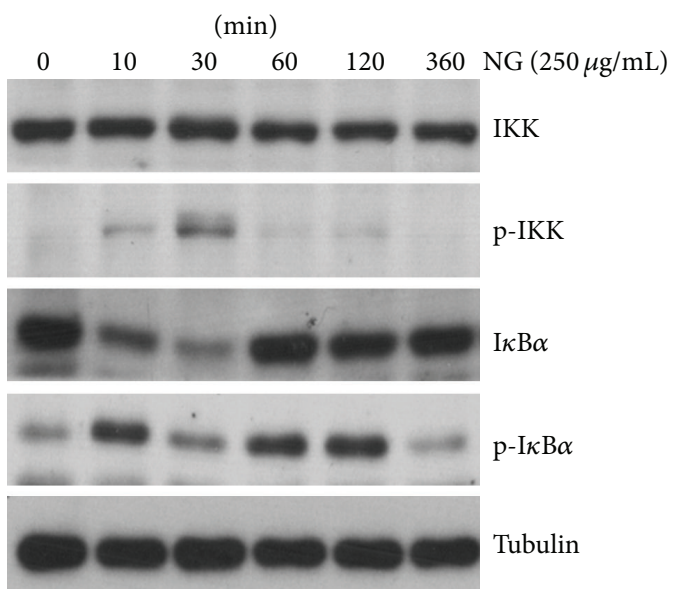

(b)

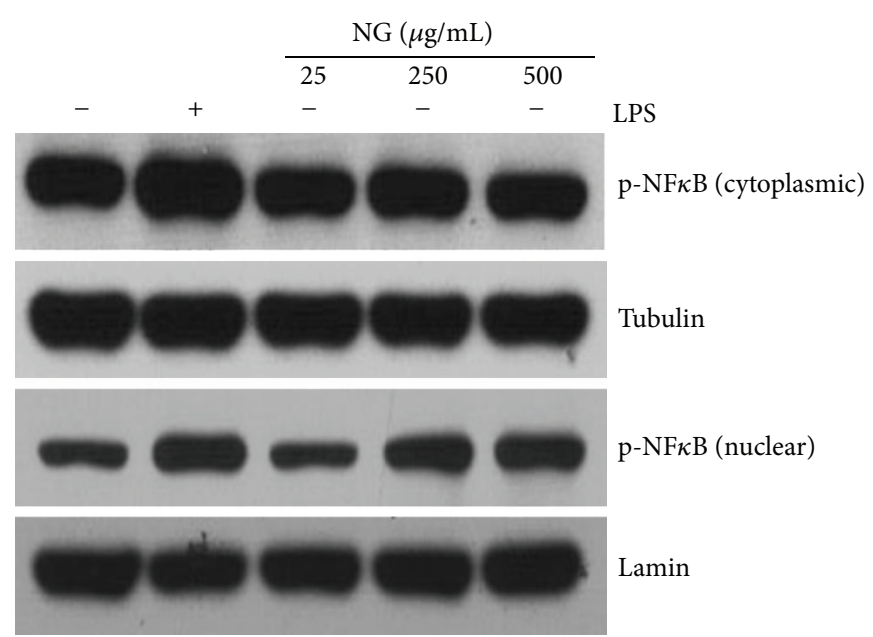

(c)

Figure 4: NG induced activation of MAPK and NF- $\kappa$ B. (a) Whole-cell lysates were analyzed by Western blot for the detection of specific proteins, as indicated (p-ERK, p-p38, p-JNK, and tubulin). (b)Whole-cell lysates were analyzed by Western blot for the detection of specific proteins, as indicated (IKK, p-IKK, I $\kappa \mathrm{B} \alpha, \mathrm{p}-\mathrm{I} \kappa \mathrm{B} \alpha$, and tubulin). (c) Nuclear and cytosolic extracts were analyzed by Western blot for the detection of specific proteins, as indicated (p-NF- $\kappa \mathrm{B}$, tubulin, and lamin).

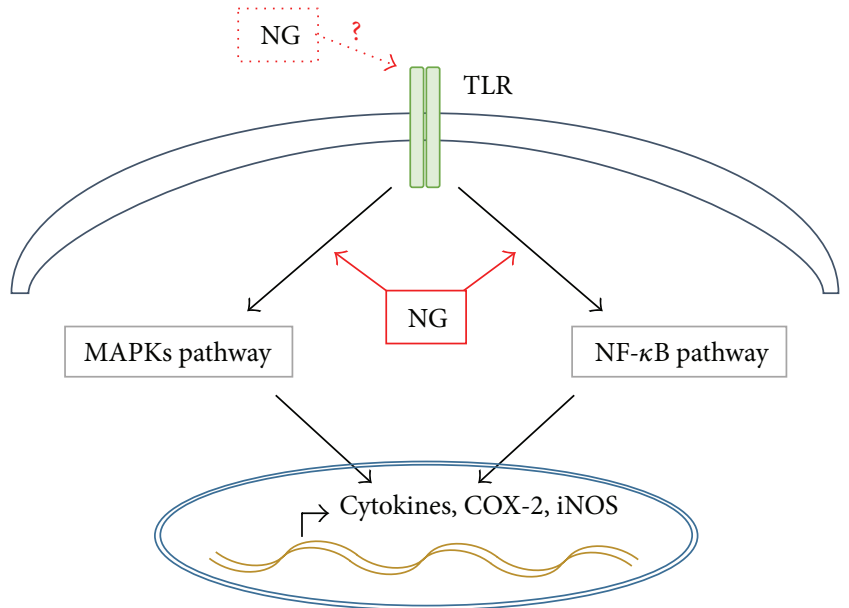

FIGURE 5: NG induces immune responses through MAPKs and NF$\kappa \mathrm{B}$ activation (schematic diagram). were diminished by approximately $30 \%$ and neutrophil numbers were increased by approximately $30 \%$. While numbers of monocytes and basophils were not altered significantly, eosinophil numbers were also significantly increased from $2.79 \%$ to $4.68 \%$. Thus, our in vivo data indicate that NG treatment may improve immune defects including neutropenia.

\section{Conclusion}

Our purpose of the study is to know whether NG has an immunomodulating effect. We confirmed that NG treatment increased ROS and promoted macrophagic differentiation of mouse monocytic Raw264.7 cells. In the in vivo study, we confirmed that NG improved immune defects including neutropenia. Hence, we conclude that NG is helpful for alleviating neutropenia. 


\section{Authors' Contribution}

H.-S. Choi and S. R. Kim equally contributed to this work.

\section{Acknowledgments}

This study was supported by Nongshim Corporation (no. 20121086), the Korea Science and Engineering Foundation (KOSEF) Grant funded by the Korea Government (MEST) (no. 2009-0063466), and a Grant from Korea Medicine R\&D Project of the Ministry of Health and Welfare (no. B120014).

\section{References}

[1] A. Safdar and D. Armstrong, "Infections in patients with hematologic neoplasms and hematopoietic stem cell transplantation: neutropenia, humoral, and splenic defects," Clinical Infectious Diseases, vol. 53, pp. 798-806, 2011.

[2] R. Sokolic, "Neutropenia in primary immunodeficiency," Current Opinion in Hematology, vol. 20, pp. 55-65, 2013.

[3] Z. Paz, M. Nails, and E. Ziv, "The genetics of benign neutropenia," The Israel Medical Association Journal, vol. 13, pp. 625-629, 2011.

[4] V. Kouranos, G. Dimopoulos, A. Vassias, and K. N. Syrigos, "Chemotherapy-induced neutropenia in lung cancer patients: the role of antibiotic prophylaxis," Cancer Letters, vol. 313, pp. 9-14, 2011.

[5] M. J. Kaplan, "Neutrophils in the pathogenesis and manifestations of SLE," Nature Reviews Rheumatology, vol. 7, pp. 691-699, 2011.

[6] M. Akhtari, B. Curtis, and E. K. Waller, "Autoimmune neutropenia in adults," Autoimmunity Reviews, vol. 9, no. 1, pp. 62-66, 2009.

[7] O. Frankfurt and M. S. Tallman, "Growth factors in leukemia," Journal of the National Comprehensive Cancer Network, vol. 5, pp. 203-215, 2007.

[8] A. W. Roberts, "G-CSF: a key regulator of neutrophil production, but that's not all!," Growth Factors, vol. 23, no. 1, pp. 33-41, 2005.

[9] G. He and M. Karin, "NF- $\kappa$ B and STAT3- key players in liver inflammation and cancer," Cell Research, vol. 21, no. 1, pp. 159168, 2011.

[10] P. S. Becker, "Growth factor priming in therapy of acute myelogenous leukemia," Current Hematology Reports, vol. 3, no. 6, pp. 413-418, 2004.

[11] M. E. Spurlock, "Regulation of metabolism and growth during immune challenge: an overview of cytokine function," Journal of Animal Science, vol. 75, no. 7, pp. 1773-1783, 1997.

[12] H. M. Lo, C. L. Chen, C. M. Yang et al., "The carotenoid lutein enhances matrix metalloproteinase- 9 production and phagocytosis through intracellular ROS generation and ERK1/2, p38 MAPK, and RARbeta activation in murine macrophages," Journal of Leukocyte Biology, vol. 93, no. 5, pp. 723-735, 2013.

[13] Y. H. Kuan, Y. C. Li, F. M. Huang, and Y. C. Chang, "The upregulation of tumour necrosis factor-alpha and surface antigens expression on macrophages by bisphenol A-glycidylmethacrylate," International Endodontic Journal, vol. 45, pp. 619-626, 2012.

[14] K. S. Janardhan, S. K. Sandhu, and B. Singh, "Neutrophil depletion inhibits early and late monocyte/macrophage increase in lung inflammation," Frontiers in Bioscience, vol. 11, no. 2, pp. 1569-1576, 2006.

[15] J. Bohlius, M. Reiser, G. Schwarzer, and A. Engert, "Impact of granulocyte colony-stimulating factor (CSF) and granulocytemacrophage CSF in patients with malignant lymphoma: a systematic review," British Journal of Haematology, vol. 122, no. 3, pp. 413-423, 2003.

[16] D. C. Dale, "Colony-stimulating factors for the management of neutropenia in cancer patients," Drugs, vol. 62, supplement 1, pp. 1-15, 2002.

[17] S. Gordon, "Alternative activation of macrophages," Nature Reviews Immunology, vol. 3, pp. 23-35, 2003.

[18] S. K. Kang, K. S. Kim, S. I. Kim, K. H. Chung, I. S. Lee, and C. H. Kim, "Immunosuppressive activity of deer antler extracts of Cervus korean TEMMINCK var. mantchuricus Swinhoe, on type II collagen-induced arthritis," In Vitro Cellular and Developmental Biology - Animal, vol. 42, no. 3-4, pp. 100-107, 2006.

[19] K. S. Kim, Y. H. Choi, K. H. Kim et al., "Protective and anti-arthritic effects of deer antler aqua-acupuncture (DAA), inhibiting dihydroorotate dehydrogenase, on phosphate ionsmediated chondrocyte apoptosis and rat collagen-induced arthritis," International Immunopharmacology, vol. 4, no. 7, pp. 963-973, 2004.

[20] Y. J. Li, T. H. Kim, H. B. Kwak, Z. H. Lee, S. Y. Lee, and G. J. Jhon, "Chloroform extract of deer antler inhibits osteoclast differentiation and bone resorption," Journal of Ethnopharmacology, vol. 113, no. 2, pp. 191-198, 2007.

[21] C. Y. Kuo, T. Wang, T. Y. Dai et al., "Effect of the velvet antler of formosan sambar deer (Cervus unicolor swinhoei) on the prevention of anllergic airway response in mice," EvidenceBased Complementary and Alternative Medicine, vol. 2012, Article ID 481318, 10 pages, 2012.

[22] T. Y. Dai, C. H. Wang, K. N. Chen et al., "The antiinfective effects of velvet antler of Formosan sambar deer (Cervus unicolor swinhoei) on staphylococcus aureus-infected mice," Evidence-Based Complementary and Alternative Medicine, vol. 2011, Article ID 534069, 9 pages, 2011.

[23] E. Szliszka, D. Skaba, Z. P. Czuba, and W. Krol, "Inhibition of inflammatory mediators by neobavaisoflavone in activated RAW264.7 macrophages," Molecules, vol. 16, no. 5, pp. 37013712, 2011.

[24] J. Ma, T. Chen, J. Mandelin et al., "Regulation of macrophage activation," Cellular and Molecular Life Sciences, vol. 60, no. 11, pp. 2334-2346, 2003.

[25] S. M. Dauphinee and A. Karsan, "Lipopolysaccharide signaling in endothelial cells," Laboratory Investigation, vol. 86, no. 1, pp. 9-22, 2006. 


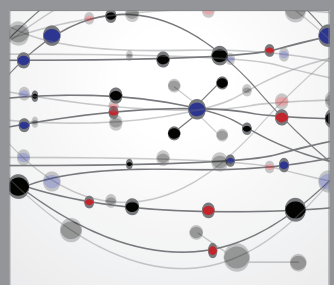

The Scientific World Journal
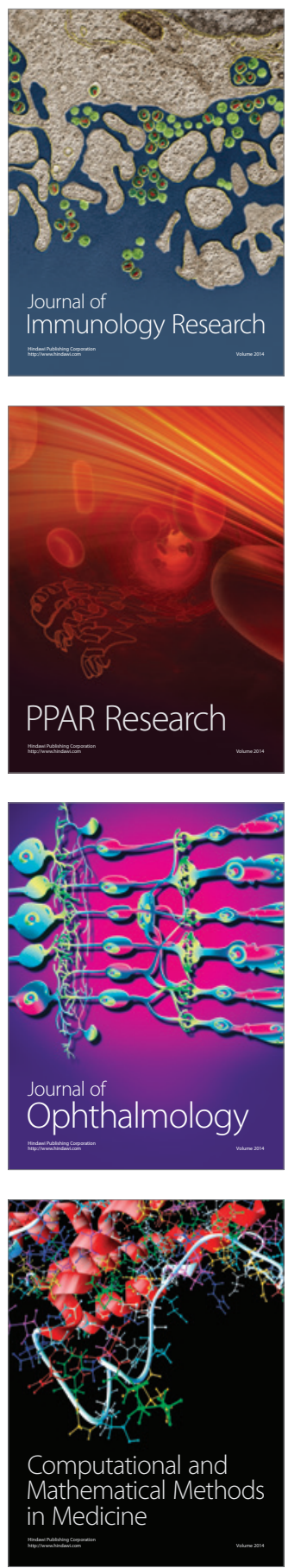

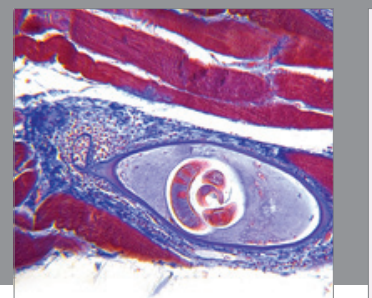

Gastroenterology

Research and Practice
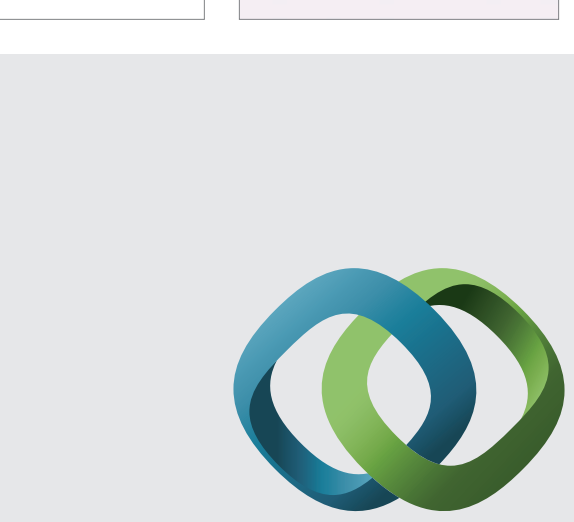

\section{Hindawi}

Submit your manuscripts at

http://www.hindawi.com
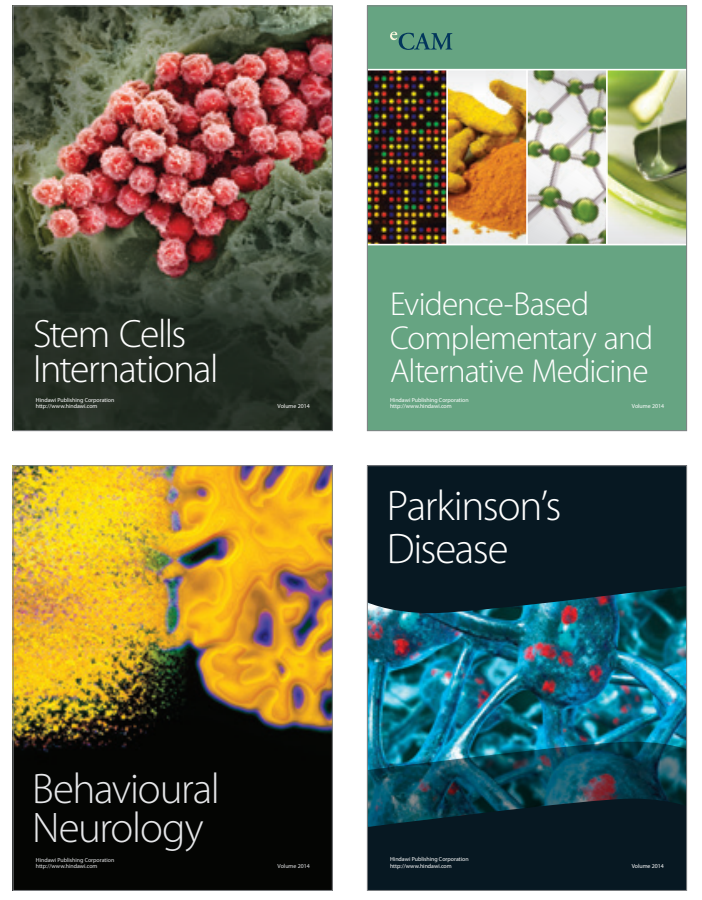
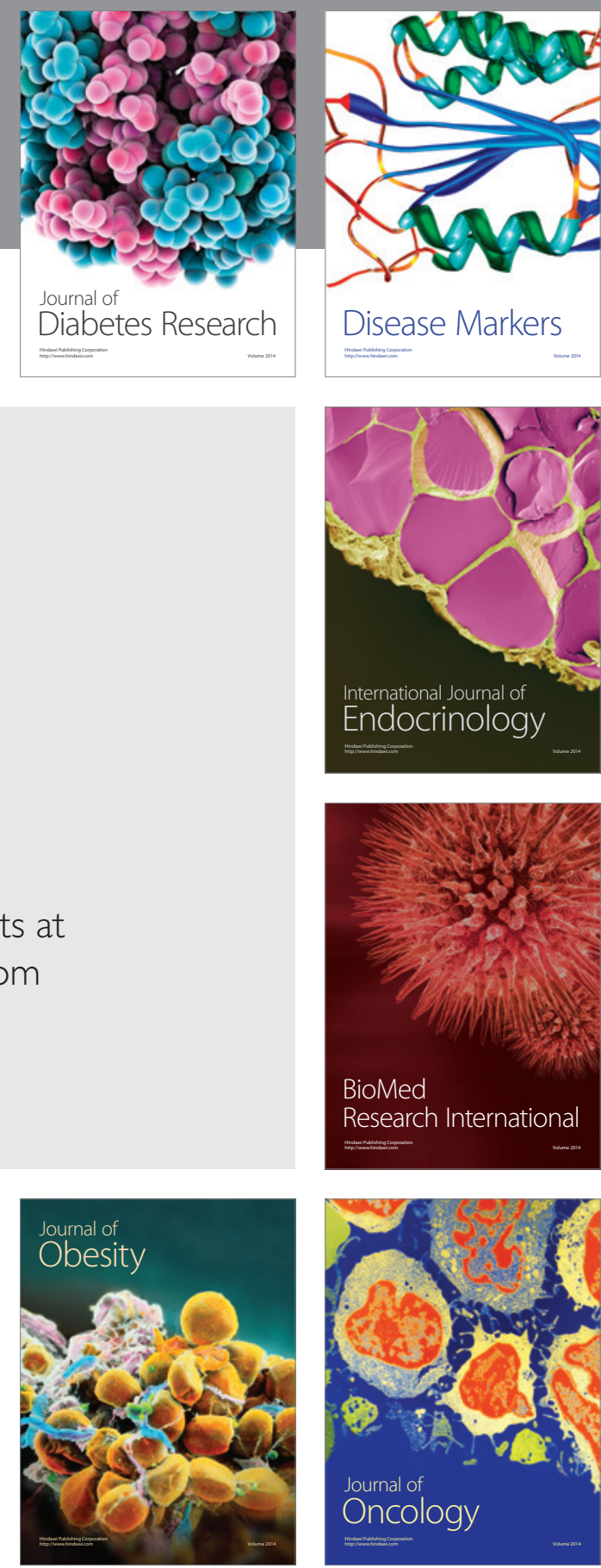

Disease Markers
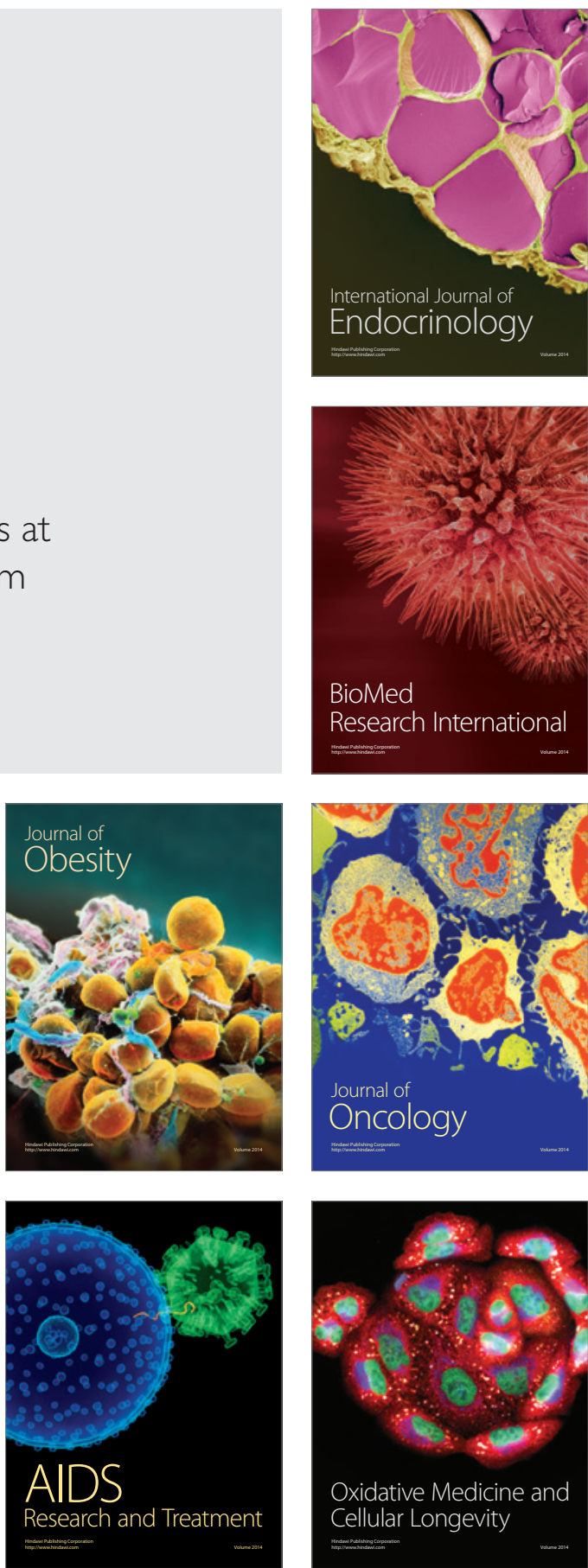